\title{
EDITORIAL
}

\section{Considerations on Impact Factor and Publications in Molecular Imaging and Biology}

\author{
Ray Gibson
}

Molecular Imaging and Biology, 115 Stoney Ford Rd., Holland, PA, 18966, USA

The impact factor (IF) for Molecular Imaging and Biology (MIB) for 2014 has been reported, 2.774. The IF is down slightly from the 2013 value of 2.869 and reflects a downward trend observed since 2011. Firstly, a definition: the IF of a journal is the average number of citations per published paper in that journal during the two preceding years. So, the impact factor for MIB for 2014 is calculated from the citations per article for the years 2012 and 2013. Although there appears to be a downward trend in citations in radiology-related journals [1], acknowledging this trend in IF for MIB prompted a vigorous discussion among the editors of the journal, of which there are 54 (3 regional editors, 10 associate editors, 18 international associate editors, and 23 members of the editorial board), to assess what the trend means and how the journal should respond to this downward trend.

One of the most interesting questions raised is whether or not we should be playing the "IF game." Some journals (e.g., American Journal of Roentgenology) have gone so far as to state that they would not play the game, but what is the significance of such a declaration? If playing the game means that one takes steps solely to increase the IF, I would heartily agree with such a stance. For example, as stated by Lee Rogers [2]:

The impact factor is also subject to deliberate manipulation. Certain types of articles, such as reviews and technical reports, are more likely to be cited than others, such as case reports and pictorial essays. To increase a journal's impact factor, editors can increase the number of reviews and eliminate case reports and pictorial essays. Editors can allow more references to be included in all articles in the belief that their journals will thereby benefit from more citations. An editor might even suggest or require that a certain number of citations be made to articles previously published in his or her journal, which will then artificially inflate the impact factor of that journal.

\section{Review Articles}

Does this mean we should not solicit some submission such as review articles? If we were to solicit an article solely because we felt it would be cited extensively, that would be problematic. However, we recognize that review articles have a purpose, which is to provide an overview of a topic that saves the reader time. We could all read the 100-150 articles that would provide us enough knowledge to be conversant, if not expert, on a topic. Or, we can read a good review article that distills the many articles into a smaller, more consumable size. Clearly, review articles provide a useful source of information, and inviting such contributions to MIB does not automatically put us in the position of playing the game solely to increase IF.

Most review articles that have been published by MIB are not solicited, but submitted to the journal much as any other article. We examine these unsolicited review articles to insure that they meet the aims and scope (A\&S) of the journal and then put them into peer review. This does not mean, however, that we should not solicit review articles. By identifying topics of interest to our readers, topics that can bring one up-to-date or to introduce background information that can benefit the reader, we serve the journal and our subscribers/members well. Thus, I strongly support the continued effort to publish one review or special topics article per issue. The difficult task is to identify appropriate review topics and appropriate authors to invite. To this end, I provide an open invitation to the members of the constituent 
societies and all other readers of MIB to consider submitting a review article to the journal. Of course, unsolicited and solicited articles are subject to peer review, so submission, even if solicited, does not guarantee acceptance.

\section{Clinical Articles}

I examined the cumulative citations for articles published in the journal over the past 3 years. This led to the observations that the most frequently cited articles in MIB are clinical papers and, as mentioned above, review articles. However, we do not want to increase our clinical component by mimicking more clinically oriented journals such as the Journal of Nuclear Medicine. Keeping within the A\&S of MIB, it is consistent and appropriate to talk to our members and colleagues about submitting their work to the journal as a first pick, not as a consolation following rejection from a so-called first-line journal. In our August issue, for example, we have five clinical papers. For our October issue, the lineup includes four clinically oriented manuscripts. I think we can all agree that the articles in the August issue are excellent papers (as are those that will appear in October), which could appear in other journals, but are nonetheless very appropriate to ours. So, my conclusion is that we should actively seek clinical contributions that fit within the A\&S of the journal.

\section{Citations-the Only or Best Metric}

One issue regarding IF is that some articles that are published in a journal receive few citations. If we did not have such under-performers, our IF would clearly be higher. I will freely admit that, as Editor-in-Chief, I cannot predict which manuscripts will be high impact, or conversely, which articles will not get cited. Some of our articles receive attention that is not reflected by the number of citations they produce. For example, two articles that have appeared over the past year have combined citations of only two. However, the same two articles are among the most frequently downloaded articles published in MIB, with combined downloads of 946. Thus, downloads are another means of assessing interest and potential impact of an article, and neither such interest nor potential impact is reflected by the IF. Perhaps citations alone are not the best way of determining potential impact of an article.

Secondly, as pointed out by Sardanelli and Sconfienza [2]:

One of the main limitations of the IF is time dependence, given that citations of articles older than 2 years do not contribute to the IF. A well-known example of this limitation is given by an article published in The Lancet in 1983 in which J. R. Warren and B. Marshall hypothesized the relationship between Helicobacter pylori and peptic ulcer. Up to
1985, the last year in which the article could have contributed to the IF of The Lancet, that article was cited 37 times. In the following years, the same article was cited more than 2000 times: Warren and Marshall won the Nobel Prize in Physiology or Medicine in 2005 for their theory, but The Lancet was not able to benefit from publication of that article.

This is observed for citations in MIB in which 2012 and 2013 citation numbers are much higher than those in 2014. Not only does more time lead to an accumulation of citations, but the more novel and interesting articles may not be accepted as quickly (e.g., the article mentioned above) or may lead to additional work/publications that take several years to mature.

The issue regarding under-cited articles raises the most important factor in our considerations - we are a peerreviewed journal. If an article is submitted and fits the $A \& S$ of the journal, then we put it into review. When the combined wisdom of our reviewers, augmented by editorial overview, dictates that a manuscript is suitable for publication and should be accepted, I find it difficult to reject an article at that time. This is not to say that reviewers are infallible. But, during my 5 years as EIC, I have only had several manuscripts for which I found the reviewers either missed a fatal flaw, or conversely, the reviewers rejected an article for reasons not well founded. So, our content, whether well cited or not, reflects the interests of colleagues who submit to MIB supported by peer review and with editorial support as needed.

\section{Who We Are}

MIB has a specific niche as defined in by our A\&S. Although we can always change the A\&S, they have served us well and reflect the overall nature of the World Molecular Imaging Society (WMIS), European Society of Molecular Imaging (ESMI), and Federation of Asian Societies of Molecular Imaging (FASMI). How do we compare to other journals serving the same niche? We have better IF than Nuclear Medicine and Biology (NMB) and Molecular Imaging (MI). The ranking of $\mathrm{MIB}>\mathrm{NMB}>\mathrm{MI}$ has been consistent for the several years. We should not be sanguine about this since we are lower than the Journal of Nuclear Medicine and the European Journal of Nuclear Medicine and Molecular Imaging, both of which still publish preclinical studies. I believe the relative rankings reflect the tendency for authors to submit their original articles to the journal with the highest impact first, and if not accepted, then to a journal that ranks lower on the IF scale. In fairness, authors are encouraged to submit their high-impact articles to journals with the highest IF to improve their evaluations by their institutions. Increasing IF is a natural consequence of having good, substantive papers submitted to our journal. This suggestion was made more than once in our editorial 
discussions and alluded to above: our membership, and as much as we can influence, our colleagues, should be encouraged to submit their articles to MIB first.

Should we play the IF game? No. However, I believe recognizing that publishers use IF in their decision on resource allocation should not be ignored. The IF, however flawed it may be, remains an important metric for assessing journal usage. Using knowledge of our IF and relative position in the ranking of journals can be used to guide us to improvements in our journal, which we undertake not specifically to target an increase in IF, though the end result may be the same.

\section{Conclusion}

We will continue largely as we have. We will continue to solicit and publish review articles with the intent to inform our readers. We ask the membership of WMIS, ESMI, and
FASMI to consider MIB first when submitting articles, though keeping in mind our specific niche as defined by our A\&S. This may result in an increase in clinical papers, but only those that also fit our A\&S; and, all this conducted within the respected methods of a peer-reviewed journal.

Acknowledgments. I thank all of the editors of MIB for their invaluable thoughts and contributions to the conversion that led to this editorial. In addition, I thank Dr. Ackerman, Dr. Gelovani, and Lorraine Gibson for helpful comments in preparing/editing this contribution.

Conflict of Interest. The author declares that he has no competing interests.

\section{References}

1. Sardanelli F, Sconfienza LM (2013) Declining impact factor of radiologic journals: a matter for debate. Am J Roentgenol 201:W391W393

2. Roger L (2002) Impact factor: the numbers game. Am J Roentgenol $178: 542-551$ 